\title{
الوضع الراهن للإنتاج العالمى للوقود الحيوى
}

\author{
ابراهيم صديق على ، عبد الرحمن محمد تريل ، صبحى أحمد أبو النجا ، \\ محمود محمد عبد المنعم ، شادى محمد شوقى عبد الموجود \\ قسم الاقتصاد الزراعى، كلية الزراعة، جامعة المنوفية
}

Received: Jan. 6, 2018

Accepted: Jan. 10,2018

الملخص:

تناول هذا هذا البحث الوضع العالمى الراهن للوقود الحيوى من حيث تطور الإنتاج من الوقود الحيوى بصفة عامة، حيث تبين أن متوسط الإنتاج العالمى من الوقود الحيوى قد بلغ نحو 14.6 مليار جالون سنوياً خلال الفترة $1991-$ 2015، كما تم دراسة تطور الإنتاج العالمى للإيثانول والذى تبين منه أن الإنتاج العالمى من الإيثانول قد بلغ فى المتوسط نحو 12 مليار جالون خلال نفس الفترة بمعدل نمو بلغ قرابة 11\% سنوياً، وكذلك تم دراسة التطور فى الإنتاج العالمى للديزل الحيوى، والذى أوضح أن متوسط الإنتاج العالمى من الديزل الحيوى قد بلغ نحو 2.4 مليار جالون بمعدل نمو بلغ نحو 15\% خلال نفس الفترة، كما تناولت الدراسة الأهمية النسبية لإنتاج الديزل الحيوى من جملة إنتاج الوقود الإدي الحيوى، حيث تبين أنها بلغت قرابة 5\% خلال نفس الفترة، وكذلك تبين انخفاض دور فول الصويا فى إنتاج الديزل الحيوى على المستوى العالمى على النحو السابق الإثارة إليه فبالتالى لا يمثل الإنتاج العالمى للايزل الحيوى من فول الصويا أثراً على المقتصد المصرى، وكذلك تبين ارتفاع دور الذرة والسكر فى إنتاج الايثانول على المستوى العالمى على الإنى النحو السابق الإثارة إليه فبالتالى يمثل الإنتاج العالمى من الايثانول من الذرة والسكر أثراً على المقتصد المصرى.

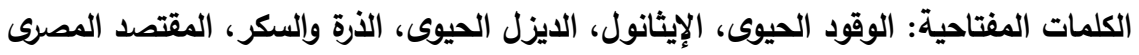

النهوض بالقطاع الزراعى وتنمية المجتمعات الريفية من

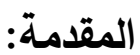
خلال إيجاد فرص للتشغيل وتوليد الاخول وتجنب هجر الأراضي والهجرة إلى المدن، مع إمكان تأمين الحصول فئل

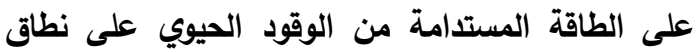

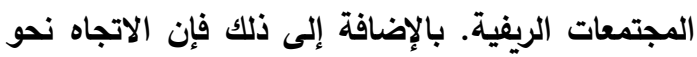

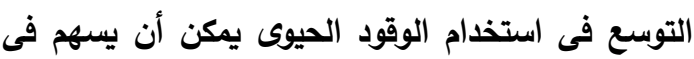

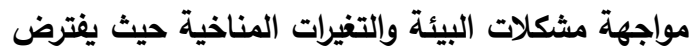
أن يحقق إمكانية خفض دورة الانبعاثات ذات الأثر السلبي في البيئة بتكلفة أقل من تلك المرتبطة بخيارات دون الانباتات دات الاتر أخرى مثل الطاقة الثمسية. ونظرا لعدم توافر بيانات إنتاج الديزل الحيوى قبل عام 1991 حيث لم يبلأ الإنتاج التجارى لله إلا منذ ذلك العام، وعليه سيتم تتاول تطور الإنتاج العالمى من الوقود الحيوى بثقيه

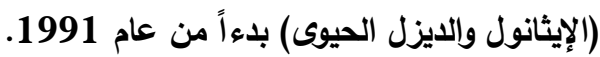

1- دينا جلال، إنتاج الوقود الحيوى في إطار الاقتصاد العالمى مع إشارة

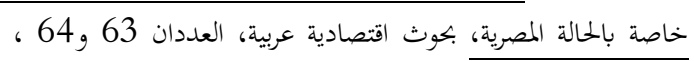
مركز دراسات الوحدة العربية، بيروت، 2013 


\section{S. Ali, et al.,}

ديزل حيـوى) قد بلـغ نحو 14.6 مليـار جـالون سنوياً خلال الفترة ( 2015-1991) وقد تراوح هذا المتوسط بين حد أدني بلغ نحو 4.22 مليار جالون عام 1992 مثل نحو 29\% من متوسط تلك الفترة، وحد أعلي بلغ بلغ دالغ نحو 37 مليار جـالون مثل نحو 256\% وذلكـ عام .2010

لقد تعرض الإنتاج العالمى من الوقود الحيوى لقدر كبير من التذبذب وعدم الاستقرار يمكن تأكيده من قيمة معامل الاختلاف البالغة حوالي 86\% خلال فترة الاراسة، ويثير الثكل الانتشارى رقم (1) إلى أنسه يمكن تقسيم

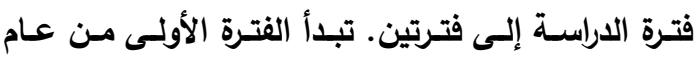
1991 إلى عام 2002 وقد اتسمت بالاستقرار النسبى

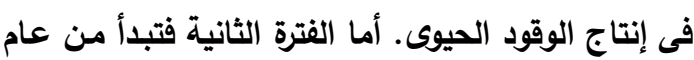
2003 وحتـى عـام 2015 والتـى اتســت بالزيــادة المضـطردة فـى إنتـاج الوقـود الحيـوى وبـإجراء اختبـار معنوية الفرق بين معاملات الانحدار للفترتين تبين وجود

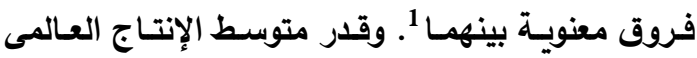
للوقود الحيوى للفترة الأولسى بنحو 4.9 مليـار جـالون، بحد ادنى بلغ 4.2 مليار جالون عام 1992 مثل نحو 86 مـن المتوسط وحد أعلى بلـغ نحو 5.6 مليـار جـالون عـام 1997 مثل نحو 115\% مسن المتوسط، وتثـير تقديرات تلـك المعادلـة إلى أن نحـو 57\% مـن التغيرات في حجم الإنتاج العالمى من الوقود الحيوى لفترة

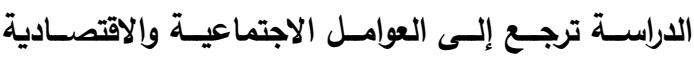
والتكنولوجية المرتبطة بالزمن، كما تشير تقديرات المعادلة إلى أن معدل النمو السنوى للإنتاج العالمى من الوقود الحيوى خلال تلك الفترة يقدر بنحو 2.2\% سنوياً.

1 - تم اختبار معنوية الفرق بين معاملات الانحدار للفترتين

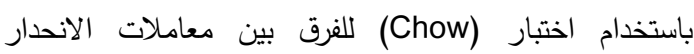

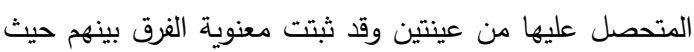
قدرت قيمة (f) المحسوبة بنحو 22.5.
مشكلة البحث: - مش

شكل النمو الكبير والمتسارع في إنتاج الوقود الحيوي حدثاً هاماً على الساحة الاقتصادية العالمية، نتيجة

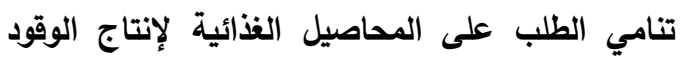

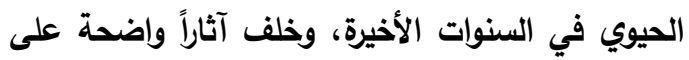

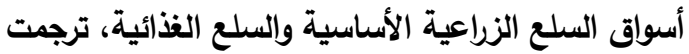

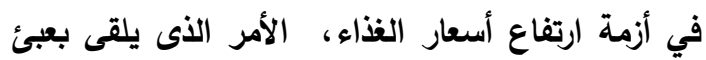

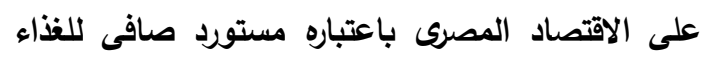
وخاصة الذرة وإلسكر.

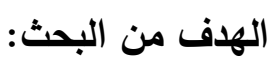
دراسة الوضع الراهن لإنتاج الوقود الحيوى فى العالم،

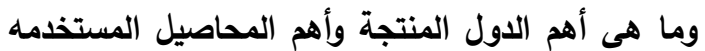

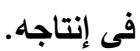
مصادر البيانات: تعتمد الاراسة بصفه رئيسية على مصادر البيانات

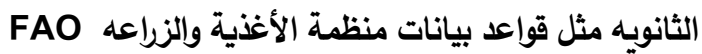
(Food Balance Sheets)، وقواعد بيانات وزارة الزراعة الأمريكية USDA، ومنظمة الدول المنتجه للبترول OPIC ودراسات المنظمات المتخصصة مثل الأل منظمة الأرض earth-policy، إتحاد منتجى الوقود الحيوى وغيرها بالإضافة إلى الرسائل والداراسات والبحوث المتاحه ذات الصلة بموضوع الدراسة. الطربقة البحثية: اعتمد البحث على استخدام أسلوب التحليل الإحصائي الوصفي وأساليب الانحار الخطى فى أثكالها

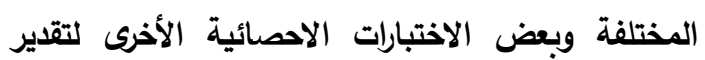
المتوسطات وكذا حساب الأهمية النسبية لانتاج الايثانول

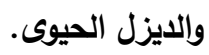
النتائج والمناقشات : أولا : تطور الإنتاج العالمى من الوقود الحيوى توضـح بيانـات الجـدول (1) أن متوســ الإنتـاج العالمى من الوقود الحيوى بمختلف أشكاله (ايثانول، 
جدول (1): الإنتاج العالمى لاجمالى الوقود الحيوى والإيثانول والديزل الحيوى ونسبة إنتاج الديزل الحيوى لاجمالى إنتاج

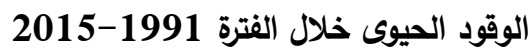

\begin{tabular}{|c|c|c|c|c|}
\hline الأهمية النسبية لإنتاج الديزل الحيوى & الإنتاج العالمى من الإيثانول & الإنتاج العالمى من الديزل الحيوى & الإنتاج العالمى من الوقود الحيوى & 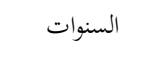 \\
\hline$\%$ & مليار جالون & مليار جالون & مليار جالون & \\
\hline 0.07 & 4.33 & 0.003 & 4.3 & 1991 \\
\hline 0.55 & 4.2 & 0.023 & 4.2 & 1992 \\
\hline 0.89 & 4.2 & 0.038 & 4.2 & 1993 \\
\hline 1.65 & 4.46 & 0.075 & 4.5 & 1994 \\
\hline 2.21 & 4.77 & 0.108 & 4.9 & 1995 \\
\hline 2.83 & 4.95 & 0.144 & 5.1 & 1996 \\
\hline 2.70 & 5.42 & 0.151 & 5.6 & 1997 \\
\hline 2.97 & 5.07 & 0.155 & 5.2 & 1998 \\
\hline 3.68 & 4.97 & 0.19 & 5.2 & 1999 \\
\hline 3.03 & 4.52 & 0.141 & 4.7 & 2000 \\
\hline 3.24 & 4.87 & 0.163 & 5.0 & 2001 \\
\hline 3.13 & 5.42 & 0.175 & 5.6 & 2002 \\
\hline 3.08 & 6.43 & 0.204 & 6.6 & 2003 \\
\hline 7.58 & 7.53 & 0.618 & 8.1 & 2004 \\
\hline 10.28 & 8.28 & 0.949 & 9.2 & 2005 \\
\hline 15.22 & 10.37 & 1.861 & 12.2 & 2006 \\
\hline 17.76 & 13.09 & 2.826 & 15.9 & 2007 \\
\hline 19.89 & 17.45 & 4.332 & 21.8 & 2008 \\
\hline 18.91 & 19.96 & 4.655 & 24.6 & 2009 \\
\hline 14.15 & 32.01 & 5.277 & 37.3 & 2010 \\
\hline 18.47 & 28.95 & 6.558 & 35.5 & 2011 \\
\hline 19.84 & 28.35 & 7.016 & 35.4 & 2012 \\
\hline 25.63 & 23.43 & 8.075 & 31.5 & 2013 \\
\hline 26.60 & 24.57 & 8.905 & 33.5 & 2014 \\
\hline 24.56 & 25.68 & 8.36 & 34.0 & 2015 \\
\hline \multirow[t]{4}{*}{5.15} & 12.1 & 2.4 & 14.6 & المتوسط \\
\hline & 32.0 & 8.9 & 37.3 & الحد الأعلى \\
\hline & 4.2 & 0.003 & 4.2 & الحد الأدنى \\
\hline & 9.6 & 3.2 & 12.6 & الانحراف المعيارى \\
\hline
\end{tabular}




\section{S. Ali, et al.,}

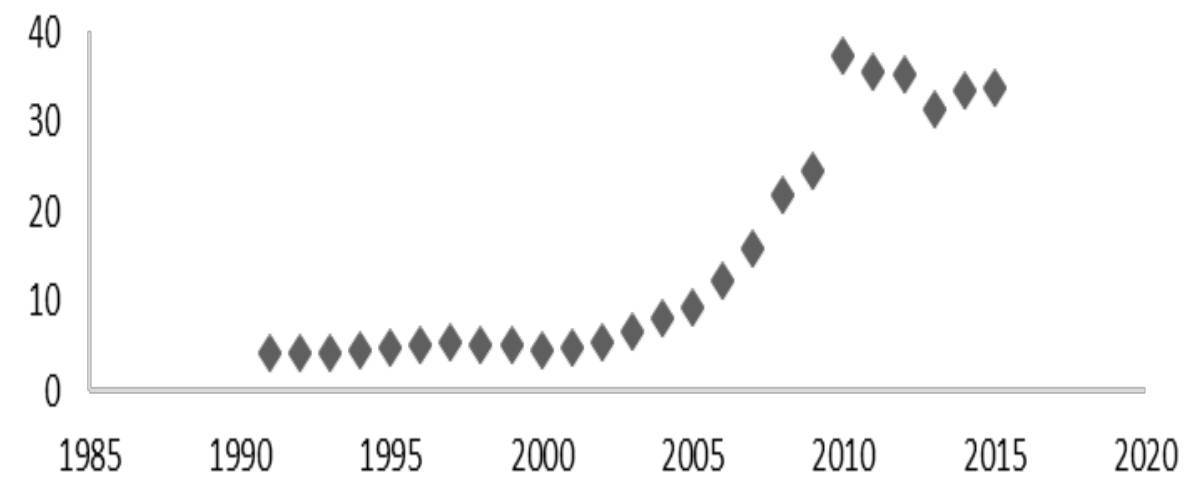

شكل رقم (1): الثكل الانتشارى للإنتاج العالمى من الوقود الحيوى خلال الفترة ( 1991 - 2015)

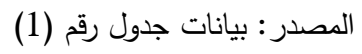

تطور الإنتاج العالمى من الإيثانول أوضحت البيانات الواردة بالجدول رقم (1) أن متوسط الإنابول

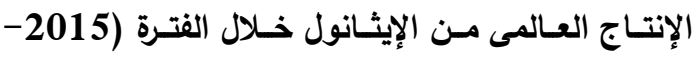
1991) قدر بنحو 12 مليـار جـالون، وقد تراوح هذا لـالئ المتوسط بين حد أدني بلغ نحو 4.2 مليار جالون عام 1992 ممثلا نحو 35\% من المتوسط، والذي أخذ في التزايـد المضطرد حتي بلغ نحو 32 مليار جالون كحد أقصى عام 2010 والذى مثل نحو 264\% من المتوسط

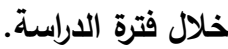

ويشـير الثـكل الانتثــارى رقـم (4) إلـى أنسه يمكن

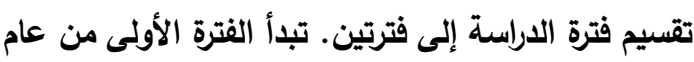

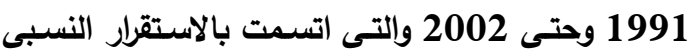
فى إنتاج الإيثانول ولكن بمعدلات منففضة، أمسا الفترة

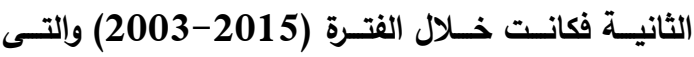
اتســت بالزيـادة السريعة فى إنتاج الإيثانول وبإبراء اختبار معنويـة الفرق 1 بين معاملات الانحدار للفترتين تبين وجود فروق معنوية بينهما.

1 - تم اختبار معنوية الفرق بين معاملات الانحدار للفترتين باستخدام اختبار (Chow) للفرق بين معاملات الانحدار المتحصل عليها من عينتين وقد ثبتت معنوية الفرق بينهم حيث لاندارت قدرت قيمة (f) المسوبة بنحو 12.12
وبدراسة الاتجاه الزمنى العام للإنتاج العالمى من

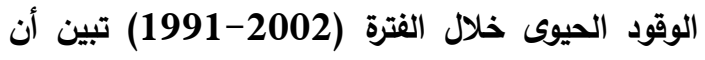

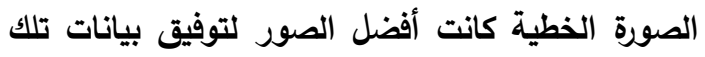

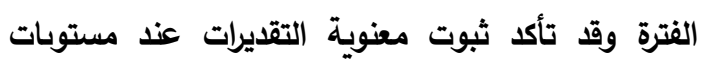
المعنوية المألوفة على النحو الذى تونى تعكسه المعادلة

بالثكل رقم (2). فى نفس الوقت قرر متوسط الإنتاج العالمى للوقود الحيوى للفترة الثانية بنحو 23.5 مليار جالون، بحد ادنى بلغ 6.6 مليار جالون مثل نحو 28\% من من ملئو

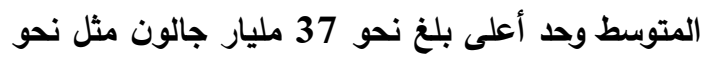
159 من المتوسط، وبدراسة الاتجاه الزمنى العام للإنتاج العالمى من الوقود الحيوى خلال الفترة (20021991) تبين أن الصورة الأسية كانت أفضل الصور الصورد لتوفيق بيانات تلك الفترة وقد تأكد ثبوت معنوية التقديرات عند مستوبات المعنوية المألوفة على النحو الذى تعكسه تاكي المعادلة بالثكل رقم (3). وتثير تقديرات تلك المعادلة إلى أن نحو 85\% من التغيرات في حجم الإنتاج العالمى من الوقود الحيوى لفترة

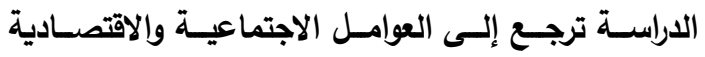
والتكنولوجية المرتبطة بالزمن، كما تثير تقديرات المعادلة إلى أن معدل النمو السنوى للإنتاج العالمى من الوقود الحيوى خلال تلك الفترة يقدر بنحو 14.9\% سنوياً. 


$$
\begin{gathered}
\hat{Y}_{i}=4.19+0.104 X_{i} \\
(19.8)^{* *}(3.64)^{* *} \\
i=1,2, \ldots \ldots \ldots \ldots, 12 \\
\mathrm{r}=0.76 \quad \mathrm{R}^{2}=0.57 \quad \mathrm{~F}=13.25
\end{gathered}
$$

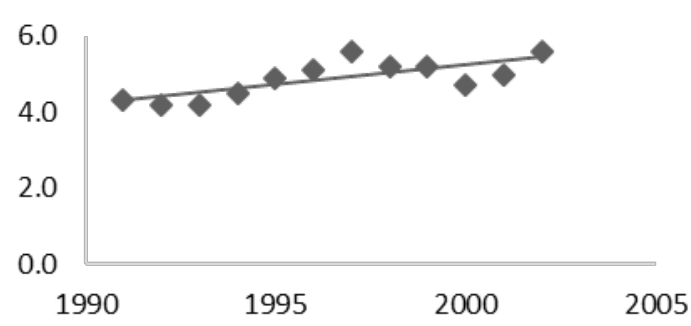

شكل رقم (2): الثكل الانتشارى للإنتاج العالمى من الوقود الحيوى خلال الفترة ( 1991- 2002)

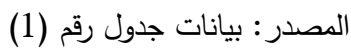

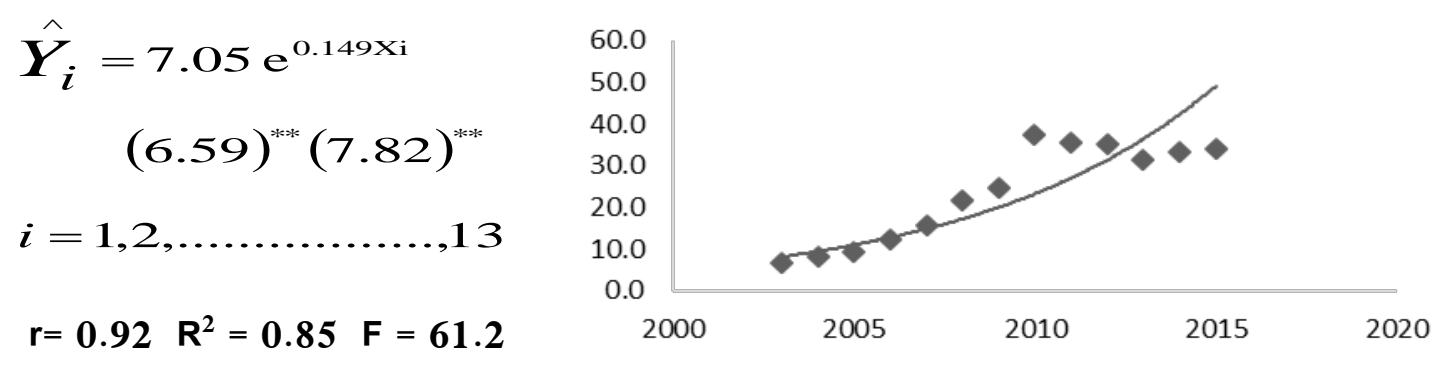

شكل رقم (3): الثكل الانتشارى للإنتاج العالمى من الوقود الحيوى خلال الفترة (2003- 2015)

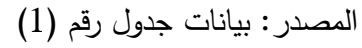

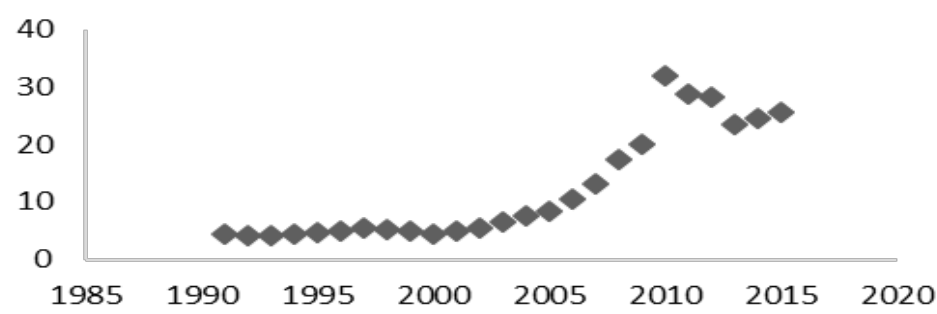

شكل رقم (4): الثكل الانتشارى للإنتاج العالمى من الإيثانول خلال الفترة (1991- 2015)

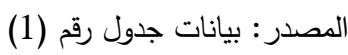

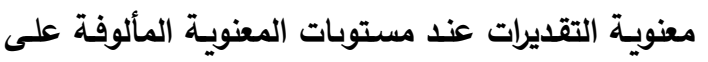

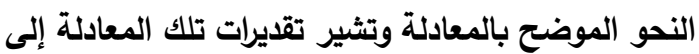

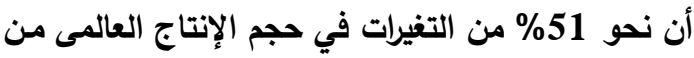

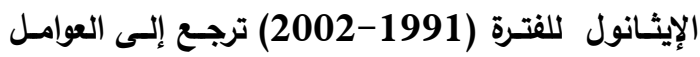
الاجتماعيـة والاقتصـادية والتكنولوجيـة المرتبطة بالزمن.

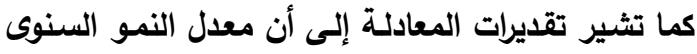

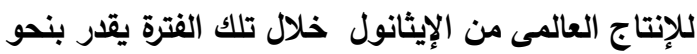

1.8 سنوياً كما فى الثكل رقم (5) الائول خلال
ويبلـغ متوسط الإنتاج العالمى مـن الإيثانول بـالفترة الأولسى نحو 4.8 مليـار جـالون بحــ أدنى 4.2 مليسار

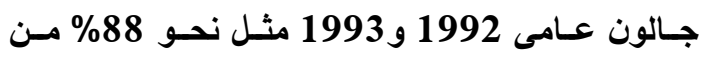
المتوسط لتلك الفترة، وحد أعلى قدر بنحو 5.4 مليار جالون عام 2002 مثل نحو 114\% من المتوسط لتلك الكانك الفترة، وبدراسـة الاتجـاه الزمنـى العـام لإنتـاج الإيثانول العالمى خلال الفترة (2002-1991) تم استخدام العديد من الصور الرياضية حيث تبين أن الصورة الخطية كانت

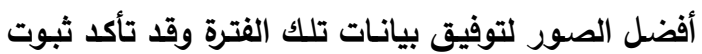




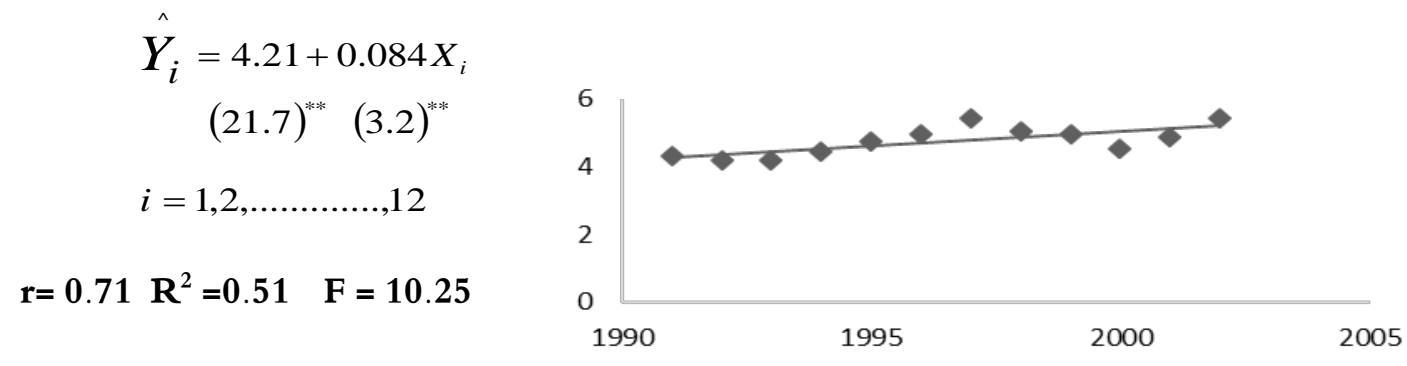

شكل رقم (5): الثكل الانتشارى للإنتاج العالمى من الإيثانول خلال الفترة (1991- 2002)

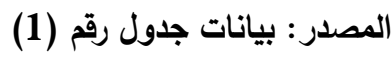

كما يشير الثكل الانتشـارى رقم (7) إلى أنسه يمكن تقسيم الفترة (2015-19915) إلى فترتين. تبدأ الفترة

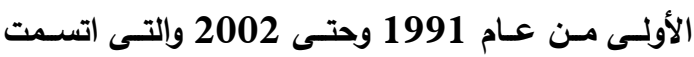
بإنتاج ضعيف وغير تجارى من الديزل الحيوى، أما الفترة

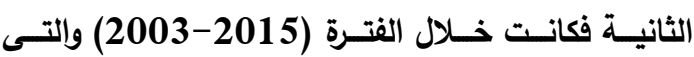

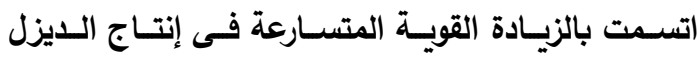

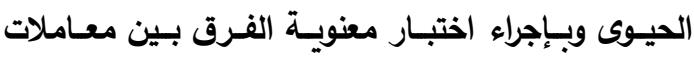

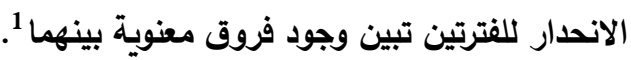

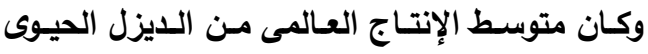

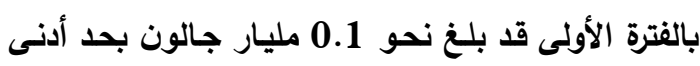

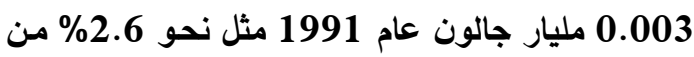
المتوسط لتلك الفترة، وحد أعلى قدر بنحو 0.2 مليار جالون عام 1999 مثل نحو 167\% من المتوسط لتلك

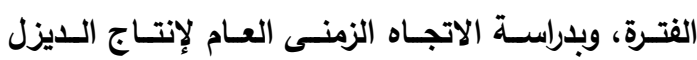

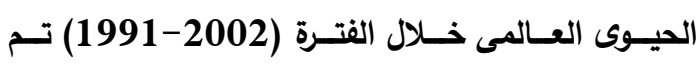

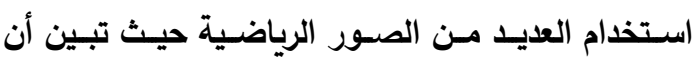

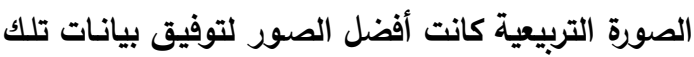

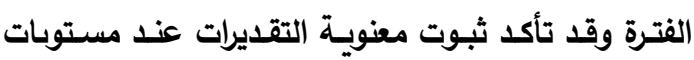

المعنوية المألوفة كما بالثكل رقم (8).

1- تم اختبار معنوية الفرق بين معاملات الانحدار للفترتين باستخدام اختبار (Chow) للفرق بين معاملات الانحدار المتحصل عليها من عينتين وقد ثبتت معنوية الفرق بينهم

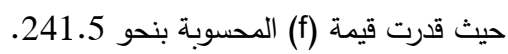

بينمـا قـدر متوسـط الإنتـاج العـالمى مسن الإيثـانول خـلال الفترة الثانيـة (2015-2003) بنحو 19 مليـار جالون بحد أعلى بلغ نحو 32 مليار جالون عام 2010 مثل نحو 169\% من المتوسط، وحد أدنى بلغ نحو 6.4 مليار جالون عام 2003 مثل نحو 34\% من المتوسط.

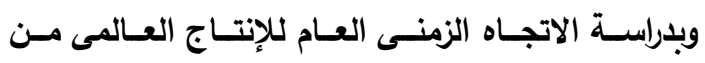

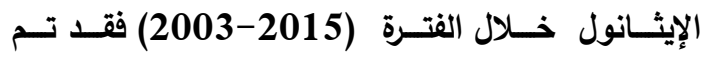

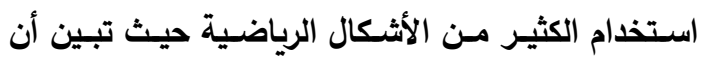

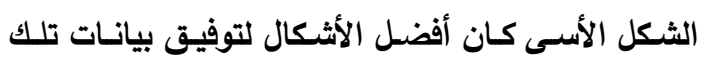

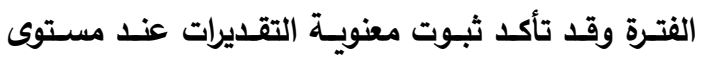

المعنوية المألوفة كما فى الثكل رقم (6). وتثير تقديرات المعادلة بالثكل رقم (6) إلى أن نحو

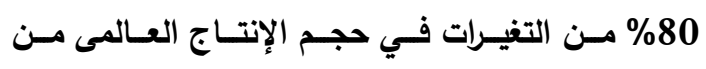
الإيثانول للفترة (2003-2015) ترجسع إلـى العوامسل

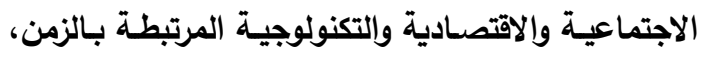
كما تثير تقديرات المعادلة إلى أن معدل النمو السنوى العائه

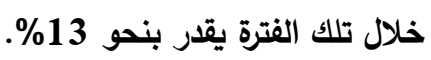

تطور الإنتاج العالمى من الديزل الحيوى

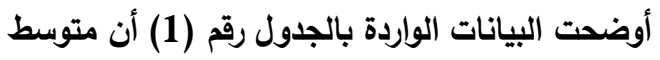
الإنتاج العالمى من الديزل الحيوى قد بلـغ نحو 2.4 مليار جالون خلال الفترة (2015-1991) قد تراوح بين الإنيز حد أقصى بلغ نحو 8.9 مليار جالون عام المن 2014 مثثلا

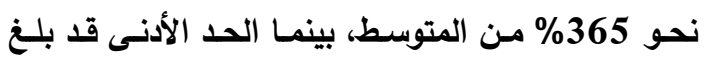
نحس 0.003 مليـار جـالون عـام 1991 والـذى مثـل نحو 0.1\% من المتوسط خلال فترة الاراسة. 


$$
\begin{aligned}
& \hat{Y}_{i}=6.7 \mathrm{e}^{0.13 \times i} \\
& \quad(6.49)^{* * *}(6.68)^{* *} \\
& i=1,2, \ldots \ldots \ldots \ldots \ldots, 13 \\
& \mathrm{r}=0.90 \quad \mathrm{R}^{2}=0.80 \quad \mathrm{~F}=44.63
\end{aligned}
$$

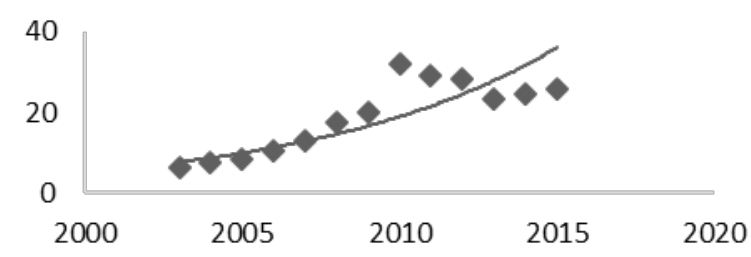

شكل رقم (6): الثكل الانتشارى للإنتاج العالمى من الإيثانول خلال الفترة (2003- 2015)

$$
\text { المصدر: بيانات جدول رقم (1) : المبكل }
$$

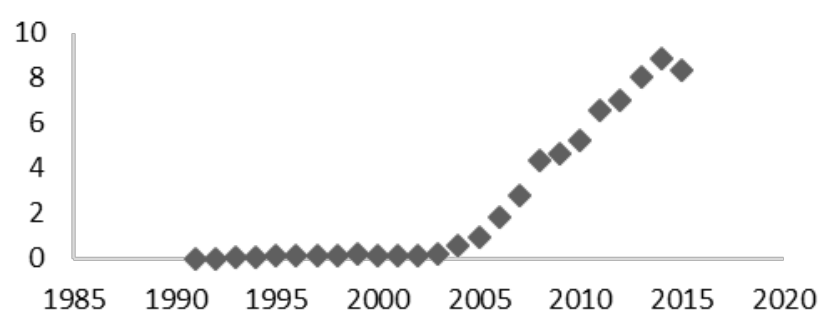

شكل رقم (7): الثكل الانتشارى للإنتاج العالمى من الديزل الحيوى خلال الفترة ’( 1991- 2015)

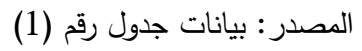

$$
\begin{gathered}
\begin{array}{c}
\hat{Y}_{i}=-0.05+0.041 X_{i}-0.0019 X_{i}^{2} \\
(-2.92)^{* * *}(6.85)^{* * *}(-4.31)^{* * *}
\end{array} \\
i=1,2, \ldots \ldots \ldots \ldots \ldots \ldots \ldots \ldots ., 12 \\
\mathbf{R}^{2}=\mathbf{0 . 9 5} \quad \mathbf{R}^{2 \cdot}=\mathbf{0 . 9 3} \quad \mathbf{F}=\mathbf{7 6 . 6 4}
\end{gathered}
$$

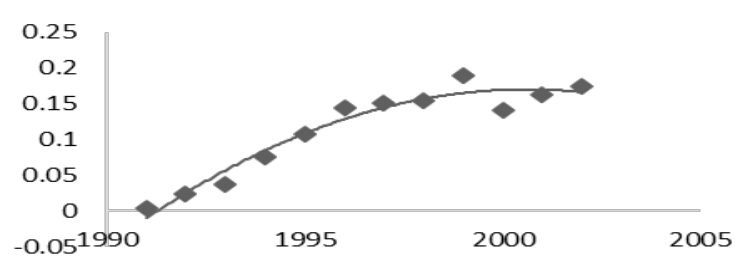

شكل رقم (8): الثكل الانتشارى للإنتاج العالمى من الديزل الحيوى خلال الفترة ( 1991- 2002)

المصدر : بيانات جدول رقم (1) : الفكل

بلـنغ نحسو 8.9 مليـار جـالون عـام 2014 مثل نحسو 194 من المتوسط، وحد أدنى بلغ نحو 0.2 مليار جـالون عـام 2003 مثل نحـو 4.4\% مـن المتوسطط، وبلراسة الاتجاه الزمنى العام للإنتاج العالمى من الديزل

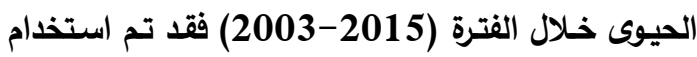
الكثير من الأثكال الرياضية حيث تبين أن الثكل الخطى (الثن كان أفضل الأثكال لتوفيق بيانات تلك الفترة وقد تأكد ثبوت معنويـة التقديرات عند مستوى المعنويـة المألوفـة على النحو الذى تعكسه المعادلة بالثكل رقم (9).
وتثير تقديرات تلك المعادلة إلى أن نحو 93\% من

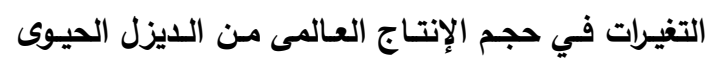
للفترة (2002-1991) ترجع إلى العوامل الاجتماعية والاقتصادية والتكنولوجية المرتبطة بالزمن، وقد أمكن من العن

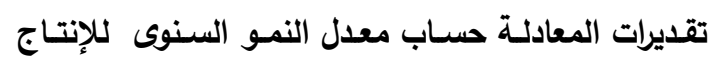
العالمى من الديزل الحيوى خلال تلك الفترة والذى قدر بنحو 2.7\% سنوياً. بينما قدر متوسط الإنتاج العالمى من الايزل الحيوى

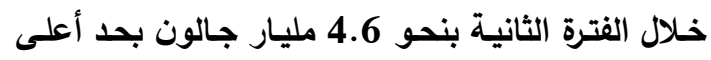




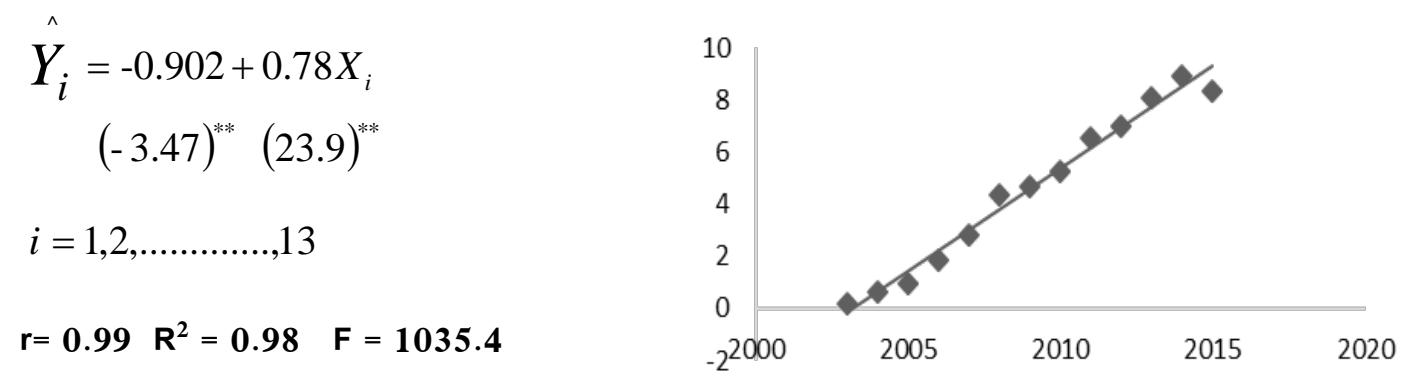

شكل رقم (9): الثكل الانتشارى للإنتاج العالمى من الديزل الحيوى خلال الفترة ( 2003- 2015)

المصدر : بيانات جدول رقم (1)

وعلى الرغم من أن المولدات والمحركات التى تعمل

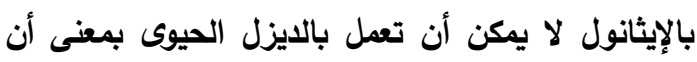

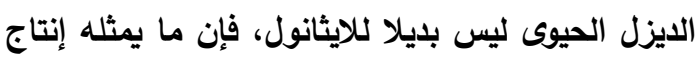

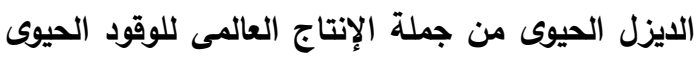
وكذلك الطاقة المتولدة عنه يمثلان قدرا ضئيلا مقارنة

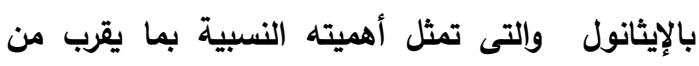
95 من الناتج العالمى من الوقود الحيوى، كما تثمل

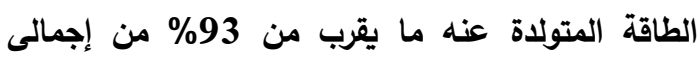
الطاقة المتولدة من إجمالى الوقود الحيوى. ويتضح من جدول رقم (3) أن إنتاج الديزل الحيوى يتركز أساسا فى كل من الاتحاد الأوروبى والولايات

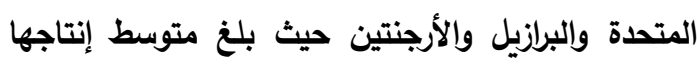

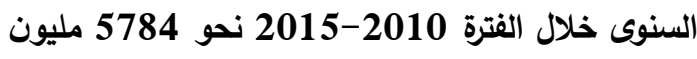
جالون تمثل حوالى 80\% من متوسط الإنتاج العالمى خلال نفس الفترة والذى يقدر بحوالى 7230 مليون جالون سنويا. ويعد الاتحاد الأوروبى أكبر منتج للديزل

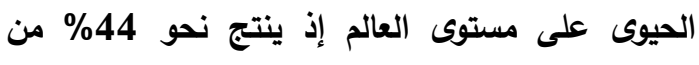
الإنتاج العالمى ويليه فى الأهمية كل من البرئ لبرازيل والأرجنتين بنسبة 16\%، 11\% على الترتيب. ومن المعروف أن الديزل الحيوى ينتج من العديد من مصادر

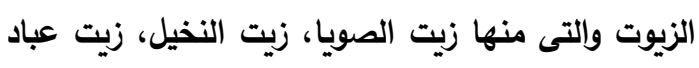

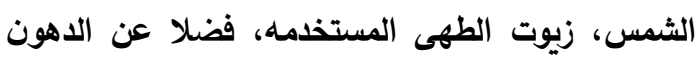

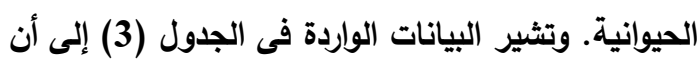
زيت فول الصويا لا يسهم سوى بنحو 27\% من الإنتاج
وتثير تقديرات تلك المعادلة إلى أن نحو 98\% من

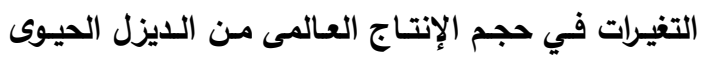
للفترة (2015-2003) ترجيع إلى العوامـل الاجتماعيـة

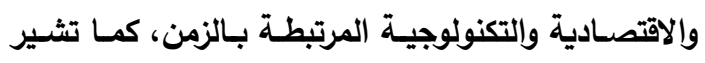
تقديرات المعادلـة إلى أن معدل النمـو السنوى للإنتاج

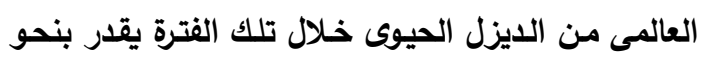
27.5 سنوياً.

الأهمية النسبية لإنتاج الديزل الحيوى من جملة إنتاج الوقود الحيوى الاهميه

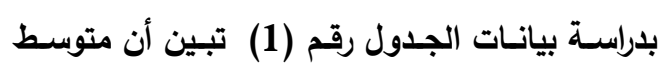

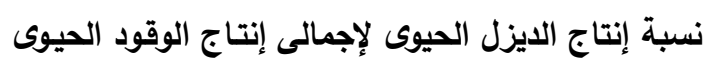

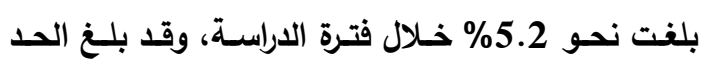
الأنسى لها عام 1991 والذى قدر بنحو حين بلغ الحد الأقصى نحو 27\% الأنى لهام ومن ناحية أخرى تثير بيانات الجدول رقم (2) أنه

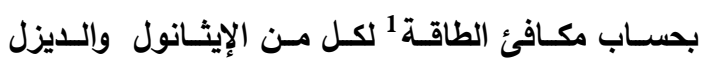
الحيوى خلال الفترة (2015-1991) أن متوسط نسبة الطئة

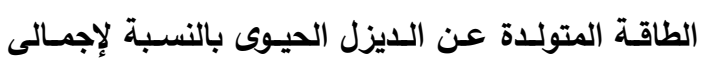

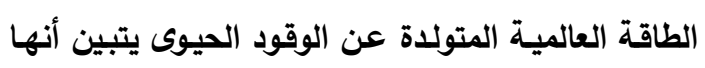
تمثل نحو 7.33\% من إجمالى الطاقة العالمية.

1 - مكافئ الطاقة: مقدار ما تساويه وحدة القياس بالطاقة 
جدول رقم (2): نسبة الطاقة المتولدة عن الايزل الحيوى لإجمالى الطاقة المتولدة عن الوقود الحيوى

\begin{tabular}{|c|c|c|c|c|c|c|}
\hline الأهية النسبية للطاقة & إجمالى الطاقة المتولدة عن & مكافئ الطاقة2 للديزل الحيوى & مكافئ الطاقة' للإيثانول & الإنتاج العلمى & الإنتاج & السنوات \\
\hline المتولدة من الديزل & الوقود الحيوى & & & من الديزل & العلمى من & \\
\hline الحيوى & & & & الحيوى & الإينانول & \\
\hline$\%$ & مليون ميجا/جول & مليون ميجا/جول & مليون ميجا/جول & مليون جالون & مليون & \\
\hline & & & & & جالون & \\
\hline 0.10 & 387055.8 & 386.8 & 386669 & 3 & 4330 & 1991 \\
\hline 0.82 & 378154.2 & 3094.2 & 375060 & 23 & 4200 & 1992 \\
\hline 1.32 & 380088.1 & 5028.1 & 375060 & 38 & 4200 & 1993 \\
\hline 2.44 & 408228.6 & 9950.6 & 398278 & 75 & 4460 & 1994 \\
\hline 3.26 & 440306.8 & 14345.8 & 425961 & 108 & 4770 & 1995 \\
\hline 4.16 & 461233.1 & 19198.1 & 442035 & 144 & 4950 & 1996 \\
\hline 3.98 & 504047.9 & 20041.9 & 484006 & 151 & 5420 & 1997 \\
\hline 4.36 & 473390.7 & 20639.7 & 452751 & 155 & 5070 & 1998 \\
\hline 5.39 & 469102.0 & 25281.0 & 443821 & 190 & 4970 & 1999 \\
\hline 4.44 & 422403.1 & 18767.1 & 403636 & 141 & 4520 & 2000 \\
\hline 4.75 & 456586.3 & 21695.3 & 434891 & 163 & 4870 & 2001 \\
\hline 4.59 & 507298.5 & 23292.5 & 484006 & 175 & 5420 & 2002 \\
\hline 4.52 & 601351.4 & 27152.4 & 574199 & 204 & 6430 & 2003 \\
\hline 10.90 & 754684.8 & 82255.8 & 672429 & 618 & 7530 & 2004 \\
\hline 14.59 & 865715.9 & 126311.9 & 739404 & 949 & 8280 & 2005 \\
\hline 21.10 & 1173740.1 & 247699.1 & 926041 & 1861 & 10370 & 2006 \\
\hline 24.34 & 1545077.6 & 376140.6 & 1168937 & 2826 & 13090 & 2007 \\
\hline 27.01 & 2134874.2 & 576589.2 & 1558285 & 4332 & 17450 & 2008 \\
\hline 25.79 & 2402008.5 & 619580.5 & 1782428 & 4655 & 19960 & 2009 \\
\hline 19.72 & 3560861.7 & 702368.7 & 2858493 & 5277 & 32010 & 2010 \\
\hline 25.24 & 3458104.8 & 872869.8 & 2585235 & 6558 & 28950 & 2011 \\
\hline 26.95 & 3465484.6 & 933829.6 & 2531655 & 7016 & 28350 & 2012 \\
\hline 33.94 & 3167081.5 & 1074782.5 & 2092299 & 8075 & 23430 & 2013 \\
\hline 35.07 & 3379356.5 & 1185255.5 & 2194101 & 8905 & 24570 & 2014 \\
\hline 32.67 & 3405940.0 & 1112716.0 & 2293224 & 8360 & 25680 & 2015 \\
\hline 7.33 & & & & & & المتوسط33 \\
\hline
\end{tabular}

http:// www.ethanolrfa.org لمصدر : جمعت وحسبت من بيانات موقع جمعية الوقود المتجدد

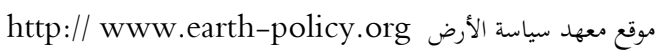
https://www.extension.iastate.edu موقع جامعة ولاية أيوض

1 gallon of ethanol $=89.3$ mega joules $-\mathrm{HHV}-1$ 1 gallon of bio-diesel $=133.1$ mega joules $-\mathrm{HHV}-2$ 3 - تم حساب الوسط الهنسى 


\section{S. Ali, et al.,}

جدول (3): التوزيع الجغرافى للإنتاج العالمى من الديزل الحيوى (مليون جالون)

\begin{tabular}{|c|c|c|c|c|c|c|}
\hline الإجمالى & أخرى & الأرجنتين & البرازيل & الولايات المتحدة الأمريكية & الاتحاد الأوروبى & 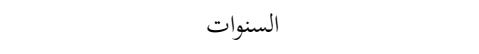 \\
\hline 5277 & 902 & 542 & 630 & 343 & 2860 & 2010 \\
\hline 6558 & 1243 & 728 & 706 & 1030 & 2851 & 2011 \\
\hline 7016 & 1570 & 737 & 718 & 1012 & 2979 & 2012 \\
\hline 8075 & 1943 & 600 & 771 & 1538 & 3223 & 2013 \\
\hline 8095 & 1248 & 776 & 903 & 1556 & 3612 & 2014 \\
\hline 8360 & 1770 & 549 & 1066 & 1411 & 3564 & 2015 \\
\hline 7230 & 1446 & 655 & 799 & 1148 & 3182 & 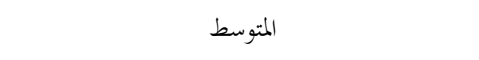 \\
\hline 100 & 20 & 9 & 11 & 16 & 44 & $\%$ \\
\hline 1970 & 48 & 59 & 575 & 1033 & 255 & متوسط الإنتاج باستخدام فول الصويا (مليون جالون) \\
\hline 27.2 & 3.3 & 9 & 72 & 90 & 8 & \% المنتجة باستخدام فول الصويا \\
\hline
\end{tabular}

مثـل الكسـافا والـــرة الثـامية والأرز والقـــح والثـعير والبطاطس والثيلم، و كذلك منها المواد السيلولوزية مثل العثـب العـوطى والميسـكانتوس والصفصـاف والحسور والعلف، ومن خـلال عملية التحويل إلى سكر والتخمير

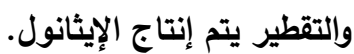

وتختلف هذه المحاصيل والمواد فيما بينها من حيث الأهمية النسبية فى إنتاج الإيثانول وكذلك تتفاوت فيما

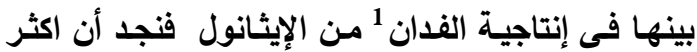
هذه المحاصيل إنتاجية هو قصب السكر بمعدل 585 جالون/فدان يليه بنجر السكر بمعدل 541 جالون/فدان، ثم تتبعهم الذرة الصفراء بمعدل 401 جالون/فدان ثماند الكسافا بمعدل 221 جالون/فئان.

بدراسة الجدول رقم (4) تبين أن نسبة مساهمة الذرة الصفراء فى إنتاج الإيثانول تمثل نحو 54\% من الإنتاج العالمى الأمر الذى يثير إلى قوة الآثار المترتبة على الإنى

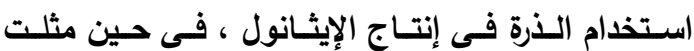

1 -D. Rajagopal et al., (2007), Challenge of Biofuel: Filling the Tank without Emptying the Stomach? Environmental Research Letters, vol. 2
العالمى للديزل الحيوى، وأن هذه النسبة تصل إلى نحو

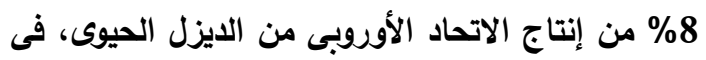

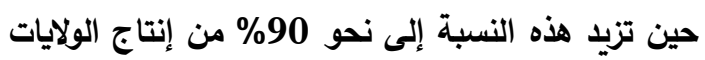

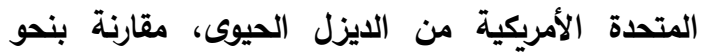
72\% بالنسبة للبرازيل، ونحو 9\%٪ 3.3 \% لكل من الأرجنتين وباقى دول العالم على الترتيب. ومن ذلك

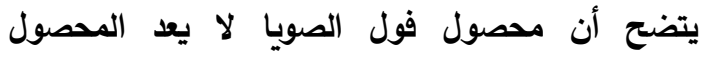
الرئيسى لإنتاج الديزل الحيوى. ولما كانت مصر من

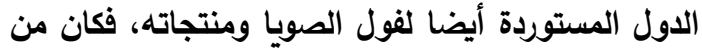
الضرورى الوقوف على أهم السلع الزراعية الداخلة فى التى إنتاج الوقود الحيوى لمعرفة أهميتها فى الإنتاج ولما

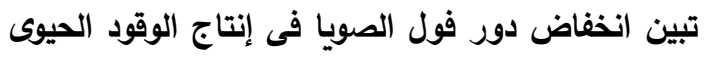
على المستوى العالمى على النحو السابق الإثارة إليه

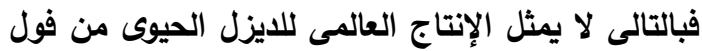
الصويا أثراً على المقتصد المصرى.

ثانيا: الأهمية النسبية للمحاصيل المستخدمة فى إنتاج الإيثانول

يدخـل العديـــ مـن أنـواع المحاصـيل فـى صـناعة الإيثانول ومنها المحاصيل السكرية مثل بنجر السكر وقصب السكر والذرة الرفيعة الحلوة، والمحاصيل النثوية 
البرازيل تصدرت الإنتاج العالمى من الإيثانول حتى عام 2004 بمتوسط إنتاج بلغ نحو 3.2 مليار جالون يمثل الإنتان لعان نحو 71\% من الإنتاج العالمى خلال الفترة (19832004)، فى حين جاءت الولايات المتحدة الأمريكية فى الآل المرتبة الثاتية خلال نفس الفترة بمتوسط إنتاج بلـغ نحو 1.3 مليـار جـالون يمثـل نــو 26 \% مسن الإنتـاج

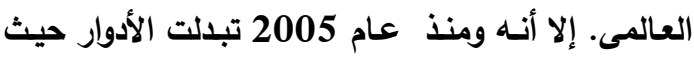
تصــرت الولايـات المتـــة الامريكيـة الترتيـب العـالمى بمتوسط إنتاج قارب 11 مليار جالون يمثل نحو 51 \% من الإنتاج العالمى بينما احتلت البرازيل المرتبـة الثانية بمتوسط إنتاج بلغ نحو 5.8 مليار جالون يمثل نحو 29

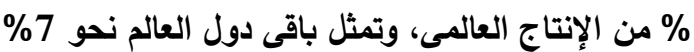
من إنتاج الإيثانول العالمى.
المحاصيل السكرية من قصب وبنجر سكر نحو 32\% من المسـاهمة في إنتاج الإيثانول العالمى بينما مثل القمح نحو 6.8\% من الإنتاج العالمى من الإيثانول ثم تأتى بـاقى المحاصيل المستخدمه فـى إنتـاج الإيثانول مجتمعسه لتمثل نسـبة نحـو 7\% مسن الإنتـاج العـالمى. ومن هنا يتضح أن الذرة والسكر يمثلان النصيب الأعظم من المساهمة فى إنتاج الإيثانول.

ثالثا : أهم الدول المنتجة للإيثانول تعد كل من الولايات المتحدة الأمريكية والبرازيل أهم الدول المنتجـة للإيثانول، ويمثل إنتاج كل من الولات الولايات

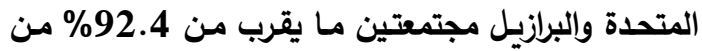

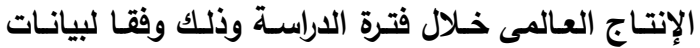
الجدول رقم (5). ويتضـح من الجدول المشـار إليه أن

جدول رقم (4): تطور الإنتاج العالمى من الإيثانول من المحاصيل الزراعية المختلفة خلال الفترة (2015-2000)

\begin{tabular}{|c|c|c|c|c|c|c|c|c|c|}
\hline \multicolumn{6}{|c|}{ 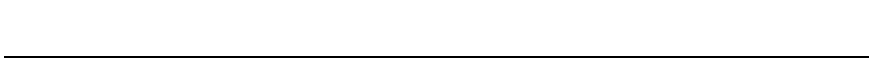 } & \multicolumn{4}{|c|}{ بالمليار جالون } \\
\hline \multirow{2}{*}{\multicolumn{2}{|c|}{ إنتاج الإيثانول من باقى المحاصيل }} & \multirow{2}{*}{\multicolumn{2}{|c|}{$\begin{array}{l}\text { إنتاج الإيثانول } \\
\text { من القمح }\end{array}$}} & \multirow{2}{*}{\multicolumn{2}{|c|}{ من المحاصيل السكرية الإيثانول }} & \multirow{2}{*}{\multicolumn{2}{|c|}{ 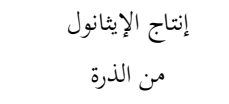 }} & \multirow{3}{*}{ من الإلتاج العالمى } & \multirow[t]{3}{*}{ 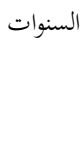 } \\
\hline & & & & & & & & & \\
\hline$\%$ & الكمية & $\%$ & 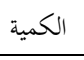 & $\%$ & 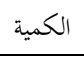 & $\%$ & 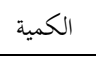 & & \\
\hline 2.2 & 0.1 & 8.3 & 0.4 & 45.7 & 2.4 & 43.8 & 2.3 & 5.2 & 2000 \\
\hline 4.2 & 0.2 & 8.0 & 0.5 & 43.0 & 2.5 & 44.9 & 2.6 & 5.8 & 2001 \\
\hline 0.1 & 0.0 & 9.6 & 0.6 & 42.2 & 2.7 & 48.1 & 3.1 & 6.4 & 2002 \\
\hline 3.8 & 0.3 & 8.3 & 0.7 & 40.5 & 3.2 & 47.5 & 3.8 & 7.9 & 2003 \\
\hline 1.2 & 0.1 & 8.5 & 0.7 & 37.9 & 3.1 & 52.4 & 4.3 & 8.3 & 2004 \\
\hline 10.5 & 1.1 & 7.8 & 0.8 & 33.3 & 3.5 & 48.3 & 5.1 & 10.5 & 2005 \\
\hline 6.8 & 0.8 & 8.4 & 1.0 & 32.2 & 4.0 & 52.5 & 6.5 & 12.4 & 2006 \\
\hline 4.5 & 0.7 & 7.2 & 1.1 & 34.3 & 5.2 & 54.0 & 8.1 & 15.1 & 2007 \\
\hline 2.2 & 0.4 & 5.7 & 1.1 & 34.6 & 6.4 & 57.5 & 10.6 & 18.5 & 2008 \\
\hline 1.9 & 0.4 & 6.4 & 1.3 & 30.4 & 6.1 & 61.2 & 12.2 & 20.0 & 2009 \\
\hline 29.2 & 9.3 & 4.5 & 1.4 & 20.1 & 6.4 & 46.2 & 14.8 & 32.0 & 2010 \\
\hline 22.7 & 6.6 & 5.0 & 1.4 & 18.2 & 5.3 & 54.2 & 15.7 & 29.0 & 2011 \\
\hline 22.1 & 6.3 & 4.8 & 1.4 & 19.1 & 5.4 & 54.1 & 15.3 & 28.4 & 2012 \\
\hline 0.6 & 0.1 & 5.7 & 1.3 & 27.7 & 6.5 & 66.0 & 15.5 & 23.4 & 2013 \\
\hline 0.4 & 0.1 & 5.5 & 1.4 & 27.2 & 6.8 & 66.9 & 16.7 & 24.9 & 2014 \\
\hline 1.2 & 0.3 & 5.3 & 1.4 & 28.0 & 7.2 & 65.5 & 16.8 & 25.7 & 2015 \\
\hline 7.1 & & 6.8 & & 32.2 & & 53.9 & & & المتوسط \\
\hline
\end{tabular}


جدول رقم (5): أهم الدول المنتجة للإيثانول ونسبتها للإنتاج العالمى بالمليار جالون خلال الفترة (2015-1983)

\begin{tabular}{|c|c|c|c|c|c|c|c|}
\hline$\%$ & باقى دول العالم & $\%$ & البرازيل & $\%$ & الولايات المتحدة الأمريكية & الإنتاج العالمى & السنوات \\
\hline 0.0 & 0.0 & 84.71 & 2.08 & 15.29 & 0.37 & 2.45 & 1983 \\
\hline 0.0 & 0.0 & 87.36 & 2.97 & 12.64 & 0.43 & 3.40 & 1984 \\
\hline 0.0 & 0.0 & 83.66 & 3.12 & 16.34 & 0.61 & 3.73 & 1985 \\
\hline 0.0 & 0.0 & 79.63 & 2.78 & 20.37 & 0.71 & 3.49 & 1986 \\
\hline 0.0 & 0.0 & 78.48 & 3.03 & 21.52 & 0.83 & 3.86 & 1987 \\
\hline 0.3 & 0.0 & 78.54 & 3.09 & 21.46 & 0.84 & 3.94 & 1988 \\
\hline 0.0 & 0.0 & 78.32 & 3.14 & 21.68 & 0.87 & 4.01 & 1989 \\
\hline 0.2 & 0.0 & 77.46 & 3.11 & 22.40 & 0.90 & 4.02 & 1990 \\
\hline 0.2 & 0.0 & 77.88 & 3.37 & 21.96 & 0.95 & 4.33 & 1991 \\
\hline 0.2 & 0.0 & 73.57 & 3.09 & 26.22 & 1.10 & 4.20 & 1992 \\
\hline 0.5 & 0.0 & 71.05 & 2.98 & 28.56 & 1.20 & 4.20 & 1993 \\
\hline 0.4 & 0.0 & 69.28 & 3.09 & 30.28 & 1.35 & 4.46 & 1994 \\
\hline 0.4 & 0.0 & 70.11 & 3.35 & 29.32 & 1.40 & 4.77 & 1995 \\
\hline 0.8 & 0.0 & 76.99 & 3.81 & 22.21 & 1.10 & 4.95 & 1996 \\
\hline 0.9 & 0.0 & 75.17 & 4.07 & 23.98 & 1.30 & 5.42 & 1997 \\
\hline 0.8 & 0.0 & 71.79 & 3.64 & 27.34 & 1.39 & 5.07 & 1998 \\
\hline 1.4 & 0.1 & 68.96 & 3.43 & 29.61 & 1.47 & 4.97 & 1999 \\
\hline 2.7 & 0.1 & 61.36 & 2.77 & 36.07 & 1.63 & 4.52 & 2000 \\
\hline 2.7 & 0.1 & 60.89 & 2.97 & 36.23 & 1.77 & 4.87 & 2001 \\
\hline 4.2 & 0.2 & 56.00 & 3.04 & 39.73 & 2.15 & 5.42 & 2002 \\
\hline 6.5 & 0.4 & 49.87 & 3.21 & 43.62 & 2.80 & 6.43 & 2003 \\
\hline 7.2 & 0.5 & 47.51 & 3.58 & 45.27 & 3.41 & 7.53 & 2004 \\
\hline 8.8 & 0.7 & 44.09 & 3.65 & 47.10 & 3.90 & 8.28 & 2005 \\
\hline 10.6 & 1.1 & 42.56 & 4.41 & 46.84 & 4.86 & 10.37 & 2006 \\
\hline 10.1 & 1.3 & 40.36 & 5.28 & 49.55 & 6.49 & 13.09 & 2007 \\
\hline 10.4 & 1.8 & 36.63 & 6.39 & 52.93 & 9.24 & 17.45 & 2008 \\
\hline 14.6 & 2.9 & 32.85 & 6.32 & 55.75 & 10.73 & 19.96 & 2009 \\
\hline 35.5 & 11.4 & 21.62 & 6.92 & 42.87 & 13.72 & 32.01 & 2010 \\
\hline 31.0 & 9.0 & 19.25 & 5.57 & 49.75 & 14.40 & 28.95 & 2011 \\
\hline 31.7 & 9.0 & 19.67 & 5.58 & 48.56 & 13.77 & 28.35 & 2012 \\
\hline 16.5 & 3.9 & 26.75 & 6.27 & 56.77 & 13.30 & 23.43 & 2013 \\
\hline 16.6 & 4.1 & 25.19 & 6.19 & 58.20 & 14.30 & 24.57 & 2014 \\
\hline 14.7 & 3.8 & 27.62 & 7.09 & 57.65 & 14.81 & 25.68 & 2015 \\
\hline \multirow[t]{4}{*}{7.0} & 1.5 & 58 & 4.0 & 35.1 & 4.5 & 10.1 & المتوسط \\
\hline & & & 7.1 & & 14.8 & 32.0 & أعلى قيمة \\
\hline & & & 2.1 & & 0.4 & 2.5 & أقل قيمة \\
\hline & & & 1.4 & & 5.2 & 9.1 & الانراف المعيارى \\
\hline
\end{tabular}


2. بودخخ كريم، حناش إلياس، أثر صناعة الوقود

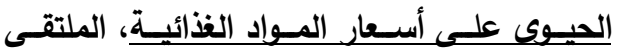

الدولى السـادس حول اشكالية الأمن الغذائى فى الغى

العـالم العربـى بجامعـة سـكيكدة، الجزائسر، 7، 8

ديسمبر 2011.

3. خالد أحمد عبده حسانين، الوضـع الحالى لإنتاج

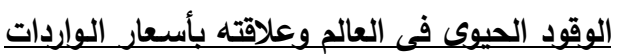

المصرية من الغذاو، مجلة المنيا للبحوث والتنمية

الزراعيـة، المجلد الرابع والثلاثون، العدد الثانى،

ديسمبر 2014.

4. دينا جلال، إنتاج الوقود الحيوى فى إطار الاقتصاد

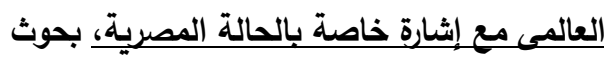

اقتصادية عربية، العددان 63 و64 ، مركز

دراسات الوحدة العربية، بيروت، 2013

5. هنـادي مصطفى عبد الراضى،|قتصـاديات الطاقـة

والغذاء؛ المؤتمر الدولى الأول لدور المواصفات

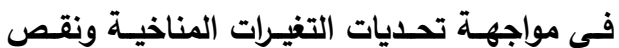

الغـذاء والميـاة والطاقة،الهيئسة المصـرية العامــة

تلمواصفات، 25/24 نوفمبر 2008.

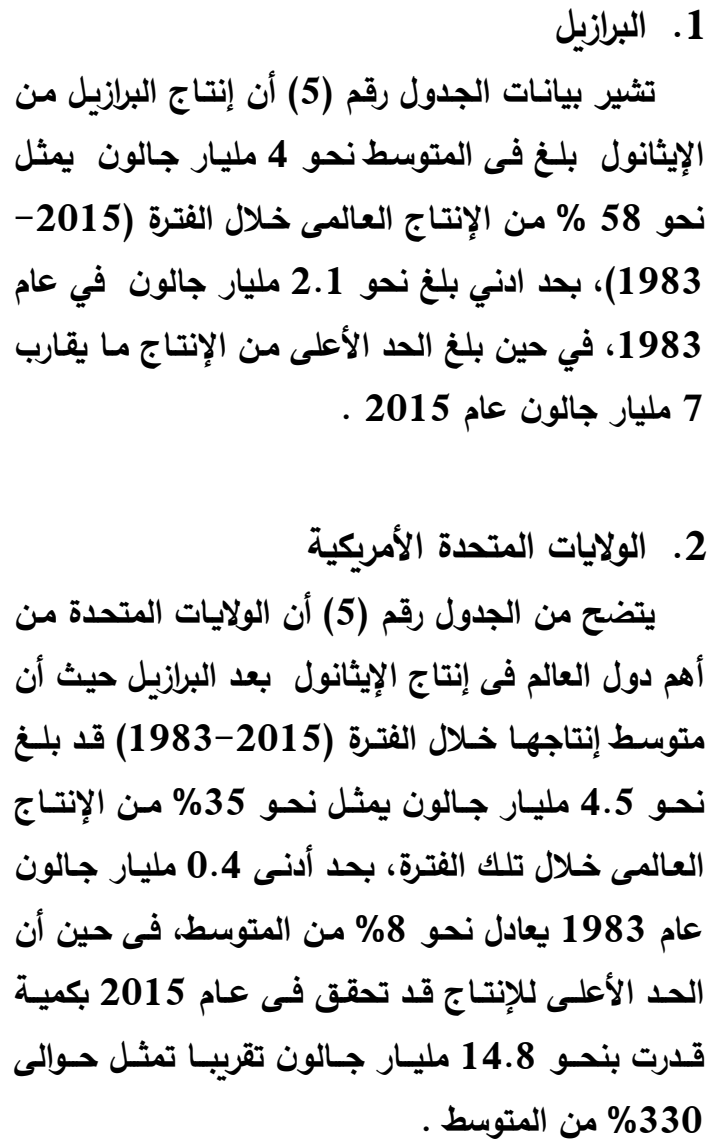

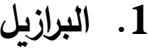

المراجع الأجنبية

1. D. Rajagopal et al., (2007), Challenge of Biofuel: Filling the Tank without Emptying the Stomach? Environmental Research Letters, vol. 2

2. Lester R. Brown, (24 January 2008), Why Ethanol Production Will Drive World Food Prices Even Higher In 2008, Earth Policy Institute.

3. http:// www.earth-policy.org

4. http:// www.ethanolrfa.org

5. http://faostat.fao.org

6. http://www.oecd.org/

7. http://www.trademap.org

8. http://www.usda.gov/psdonline/

9. https://www.eia.gov/
التوصيات:

الذرة والسكر فى انتاج الايثانول ولما كانت مصر من الدول المستوردة لكلا المحصولين فلابد من دراسة الأثر الواضح لاستخدام كل من الذرة والسكر فى انتاج الايثانول على المقتصد المصرى.

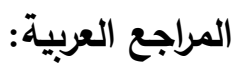

1. أمين اسماعيل عبده، خيرى حامد العشماوى، ليلى

مصطفى الثريف، رانيا محمد برغش، محددات

إنتاج الوقود الحيوى من المصـادر النباتيـة وآثاره

الاقتصادية على الواردات الغذائية المصرية، مجلة

الاقتصـاديين العرب، المجلد الثانى، العدد الأول،

2010
يتضح من الاراسة ضخامة نسبة مساهمة كلا من 


\title{
CURRENT STATUS OF GLOBAL BIOFUEL PRODUCTION
}

\author{
I. S. Ali, A. M. Taryal, S. A. Abou-El Naga and M. M. Abd-Elmoneim \\ Agricultural Economics Department, Faculty of Agriculture, Menufiya University
}

\begin{abstract}
The study examined the current global situation of biofuels in terms of the development of production of biofuels in general. The average global production of biofuels was estimated at 14.6 billion gallons annually during the period 1991-2015. The development of global ethanol production was also studied. Global production of ethanol reached in average of about 12 billion gallons during the same period at a rate of growth of about $11 \%$ per year. Also, the development of the world production of bio diesel was studied. The average global production of bio diesel reached 2.4 billion gallons at a growth rate reached about $15 \%$ during the same period, as The study examined the relative importance of the production of bio-diesel from the total bio-fuel production. It was found that it reached about $5 \%$ during the same period. Also, the role of soybeans in the production of bio-diesel has been shown at the global level as mentioned above. Soybeans have an impact on the Egyptian economy, as well as an increase in the role of maize and sugar in the production of ethanol at the global level as mentioned above. Thus, global production of ethanol from corn and sugar is an impact on the Egyptian economy.

Kay words: Biofuels, Ethanol, Bio-diesel, Maize and Sugar, Egyptian economy.
\end{abstract}

